\title{
Respiratory and circulatory deaths attributable to influenza A \& B
}

\author{
Liselotte van Asten*, Jan van de Kassteele and Wim van der Hoek \\ RIVM (Nethelands Institute of Public Health and the Environment, Centre for Infectious Disease Control), Bilthoven, Netherlands
}

\section{Objective}

To estimate mortality attributable to influenza adjusted for other common respiratory pathogens, baseline seasonal trends and extreme temperatures.

\section{Introduction}

Assigning causes of deaths to seasonal infectious diseases is difficult in part due to laboratory testing prior to death being uncommon. Since influenza (and other common respiratory pathogens) are therefore notoriously underreported as a (contributing) cause of death in deathcause statistics modeling studies are commonly used to estimate the impact of influenza on mortality.

\section{Methods}

Using primary cause of death (Statistics Netherlands) we modeled weekly timeseries of

1) respiratory deaths (ICD10 codes J00-J99) and

2) circulatory deaths (ICD10 codes I00-I99).

We used regression models with an identity link and Poisson error to relate mortality to counts of influenza A \& B diagnoses. We adjusted for other common respiratory pathogens (all pathogen data was at population level from the national laboratory surveillance), temperature (from the Dutch Royal Meteorological Institute), and baseline linear and cyclical (i.e. seasonal) trends. To account for the yearly variation in the severity of the main circulating influenza A strain we used time dependent variables for influenza A (fixed at lag 0 - assuming a direct effect of influenza. For influenza B and the confoundig pathogens we considered a 0 tot -4 time lag (thus allowing infection to precede death for up to 4 weeks). We performed the analyses separately per death cause group and by 3 different age groups (0-64, 65-74,75+ years) over a 14-year timeperiod (mid 1999-mid 2013, thus 14 complete winter seasons).

\section{Results}

In the Netherlands on average 2,636 all cause deaths occur per week varying by season (lower in summer min: 2,219 and higher in winter max: 3,564) with yearly incidence ranging from 20/10,000 in 0-64 year olds to $885 / 10,000$ in 75 -plus year olds.

Circulatory mortality ( $31 \%$ of total deaths) was higher than respiratory mortality ( $10 \%$ of total deaths) and both showed clear seasonality in all age-groups. Overall, $0.14 \%$ of all deaths were actually coded as influenza deaths.

Preliminary model estimates showed that the proportion of respiratory deaths attributable to influenza A were quite similar for 0-64 and $65-74$ year olds but higher in $75+(5.1 \%, 5.7 \%, 7.0 \%$ respectively $)$ while this proportion was stable across age-groups for circulatory deaths (approximately $1.5 \%$ in all agegroups for influenza A). Influenza B was significantly associated with respiratory deaths and circulatory deaths in the oldest age group of $75+$ years (with proportions of $0.7 \%$ and $0.2 \%$ respectively) while in the 65-74 year olds it was associated only with circulatory deaths $(0.2 \%)$. Influenza B was not significantly associated with either respiratory or circulatory mortality in the $0-64$ year age group.

On average, yearly in the $75+$ age group $70 / 10,000$ respiratory deaths and 39/10,000 circulatory deaths were attributable to influenza
A. For influenza B the incidences were 7 to 10 fold lower $(7 / 10,000$ and 6/10,000 respectively).

\section{Conclusions}

Influenza A was significantly associated with respiratory and circulatory mortality in all age groups while influenza B was significantly associated with respiratory and circulatory mortality in the elderly only.

\section{Keywords}

mortality; Influenza; Respiratory

\section{*Liselotte van Asten}

E-mail: liselotte.van.asten@rivm.nl 